\title{
Study Of Metal-Support Interactions In Model Nanocatalysts: Anchoring Of Pt Metallic Nanoparticles On Alumina Support
}

Jingyue Liu

Science \&Technology, Monsanto Company, 800 N. Lindbergh Blvd., St. Louis, MO, USA

Catalyst deactivation or the loss over time of activity and/or selectivity of a catalyst is of great concern in developing industrial catalyst; a fast catalyst that deactivates rapidly during the catalytic reaction is of little commercial value. Thus, understanding catalyst deactivation and more importantly developing strategies to suppress catalyst deactivation are of great importance. One of the intrinsic mechanisms of catalyst deactivation is sintering: the loss of active centers due to growth of the catalytic phases. For supported metal catalysts, there are three principal mechanisms of metal crystallite growth: 1) crystallite migration, 2) atomic migration, and 3) vapor transport. Regardless of the specific mechanisms sintering processes, in general, are kinetically slow but detrimentally irreversible or difficult to be reversed. Thus, one of the strategies in developing nanostructured catalysts is to prevent or significantly slow down the sintering of metal nanoparticles during the catalytic processes. Many parameters affect the growth of metal nanoparticles during the catalytic reaction: temperature, atmosphere, size of nanoparticles, metal type, surface area and tortuosity of the support, promoters and impurities, texture and porosity of the support, etc. A typical example of catalyst deactivation by sintering is the growth of noble metal nanoparticles in the three-way catalyst during the high temperature reaction in the automobile exhaust.

To understand the metal-support interaction and the sintering behavior of supported metal catalysts, we developed a model nanocatalyst consisting of Pt nanoparticles supported on polycrystalline alumina. The Pt nanoparticles were sputtered onto pretreated polycrystalline alumina slab; the model $\mathrm{Pt} /$ alumina catalysts were then reduced at higher temperatures to accelerate the sintering of $\mathrm{Pt}$ nanoparticles. We investigated the effect of the support treatment on the sintering behavior of $\mathrm{Pt}$ nanoparticles with the goal of reducing high-temperature sintering of $\mathrm{Pt}$ nanoparticles by engineering the structure of the support material.

Figure 1 shows a low-voltage SEM image of the model catalyst after high-temperature reduction. Significant sintering occurred during the reduction process as evidenced by the formation of large, faceted Pt islands. On some crystallographic faces of the polycrystalline alumina support, twodimensional Pt islands were formed (indicated by the single arrows); on other faces of the alumina support, three-dimensional Pt particles were formed (indicated by the double arrows). Detailed examination of the SEM images, however, showed that the formation of the large Pt particles had occurred only on certain surfaces of the alumina support. On alumina surfaces with high-density of steps or other surface defects, the degree of the sintering of Pt nanoparticles was significantly reduced (indicated by the letter $\mathrm{S}$ ); the surface steps anchored the Pt nanoparticles during the reduction process.

To see the effect of step anchoring of Pt nanoparticles more clearly, figure 2 shows a low-voltage SE image of another region of the model catalyst. The $\mathrm{Pt}$ nanoparticles on the alumina surface (indicated by the letter S) did not sinter much at all during the reduction process. The inset shows the high magnification image of the small Pt nanoparticles decorating surface steps or other surface defect sites. The degree of the sintering of the Pt nanoparticles is determined by the mobility of the Pt nanoparticles on the alumina surfaces, which is determined by the bonding of the Pt nanoparticles 
to the alumina support. The structure of the interfaces between the Pt nanoparticles and the alumina support critically determines the sintering behavior of the supported Pt/alumina model catalyst. Engineering of the catalyst support clearly plays an important role in reducing the sintering of supported metal catalysts. The sintering behaviors of different noble metals supported on polycrystalline alumina and their dependence on reduction temperature and support structure will be discussed.

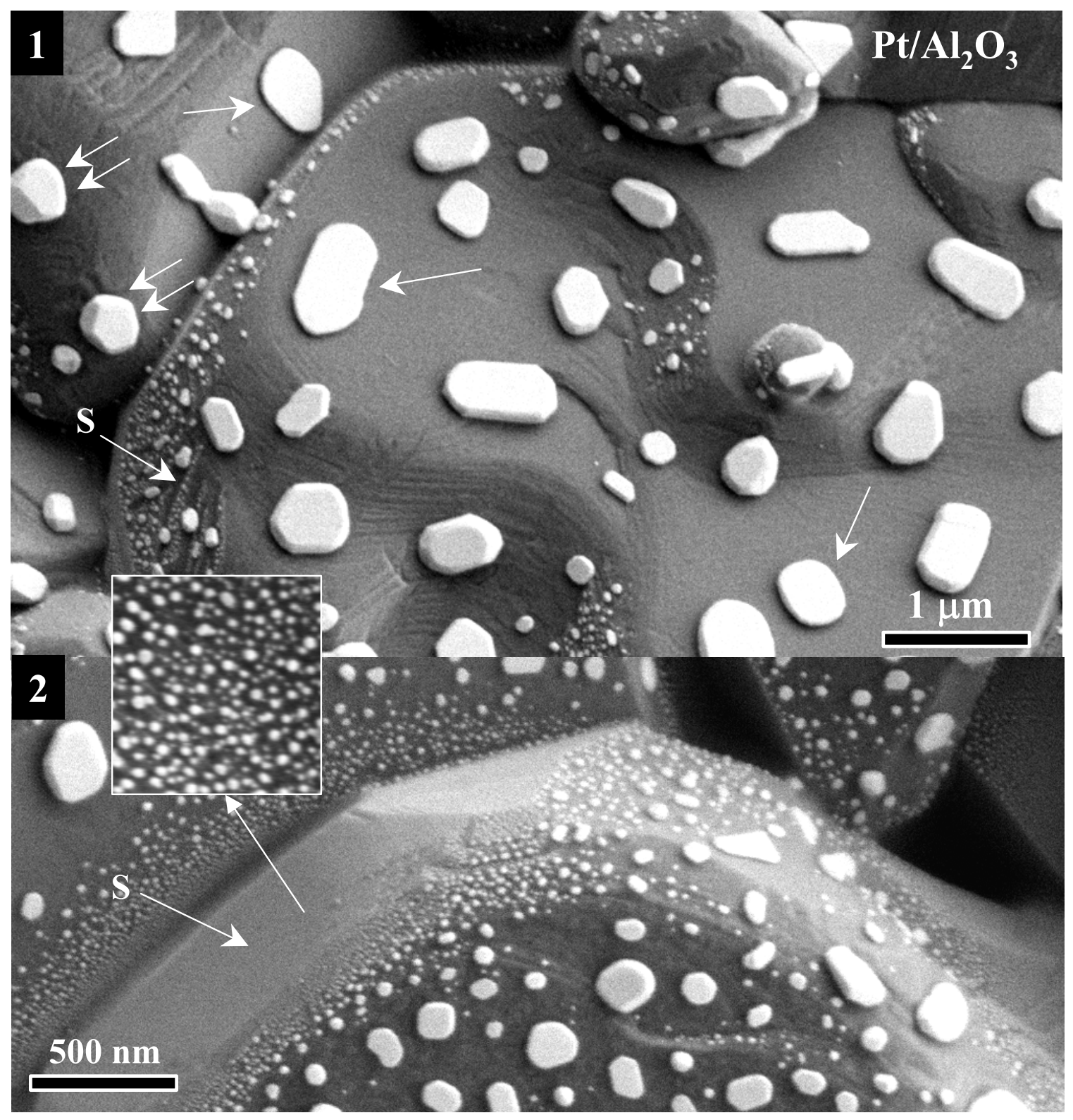

Fig. 1 Low voltage SE image of a Pt/alumina model nanocatalyst shows formation of large Pt islands due to the sintering of $\mathrm{Pt}$ nanoparticles during the reduction process and anchoring of $\mathrm{Pt}$ nanoparticles at surface steps.

Fig. 2 Low voltage SE image of a Pt/alumina model nanocatalyst shows anchoring of Pt nanoparticles on certain crystallographic faces of the polycrystalline alumina support. 